Pacific

Journal of

Mathematics

\title{
ON A CONJECTURE OF ERDÖS AND CERTAIN DIRICHLET SERIES
}

TAPAs ChatTerJee AND M. RAm Murty 


\title{
ON A CONJECTURE OF ERDÖS AND CERTAIN DIRICHLET SERIES
}

\author{
TApas ChatterjeE And M. Ram Murty
}

Let $f: \mathbb{Z} / q \mathbb{Z} \rightarrow \mathbb{Z}$ be such that $f(a)= \pm 1$ for $1 \leq a<q$, and $f(q)=0$. Then Erdôs conjectured that $\sum_{n \geq 1} f(n) / n \neq 0$. For $q$ even, it is easy to show that the conjecture is true. The case $q \equiv 3(\bmod 4)$ was solved by Murty and Saradha. In this paper, we show that this conjecture is true for $82 \%$ of the remaining integers $q \equiv 1(\bmod 4)$.

\section{Introduction}

In a written communication with Livingston, Erdős made the following conjecture (see [Livingston 1965] ): if $f$ is a periodic arithmetic function with period $q$ and

$$
f(n)= \begin{cases} \pm 1 & \text { if } q \nmid n \\ 0 & \text { otherwise }\end{cases}
$$

then

$$
L(1, f)=\sum_{n=1}^{\infty} \frac{f(n)}{n} \neq 0
$$

where the $L$-function $L(s, f)$ associated with $f$ is defined by the series

$$
L(s, f):=\sum_{n=1}^{\infty} \frac{f(n)}{n^{s}} .
$$

In 1973, Baker, Birch and Wirsing, using Baker's theory of linear forms in logarithms, proved the conjecture for $q$ prime [Baker et al. 1973, Theorem 1]. In 1982, Okada [1982] established the conjecture if $2 \varphi(q)+1>q$. Hence, if $q$ is a prime power or a product of two distinct odd primes, the conjecture is true. In 2002, R. Tijdeman [2002] proved the conjecture is true for periodic completely multiplicative functions $f$. Saradha and Tijdeman [2003] showed that if $f$ is

Research of the first author was supported by a postdoctoral fellowship at Queen's University, and that of the second by an NSERC Discovery grant and a Simons fellowship.

MSC2010: primary 11M06, 11M41; secondary 11M20.

Keywords: Erdős conjecture, Okada's criterion, nonvanishing of Dirichlet series. 
periodic and multiplicative with $\left|f\left(p^{k}\right)\right|<p-1$ for every prime divisor $p$ of $q$ and every positive integer $k$, then the conjecture is true.

It is easy to see that

$$
L(1, f)=\sum_{n=1}^{\infty} \frac{f(n)}{n}
$$

exists if and only if $\sum_{n=1}^{q} f(n)=0$. If $q$ is even and $f$ takes values \pm 1 with $f(q)=0$, then $\sum_{n=1}^{q} f(n) \neq 0$. Hence the conjecture holds for even $q$.

In 2007, Murty and Saradha [2007] proved that if $q$ is odd and $f$ is an odd integer-valued odd periodic function then the conclusion of the conjecture holds. In 2010, they proved that the Erdős conjecture is true if $q \equiv 3(\bmod 4)$ [Murty and Saradha 2010, Theorem 7]. Thus the conjecture is open only in cases where $q \equiv 1(\bmod 4)$. However, it seems that a novel idea will be needed to deal with these cases. In this paper, we adopt a new density-theoretic approach which is orthogonal to earlier methods. Here is the main consequence of our method:

Theorem 1.1. Let $S(X)=\mid\{q \equiv 1(\bmod 4), q \leq X \mid$ Erdös conjecture is true for $q\} \mid$. Then

$$
\liminf _{X \rightarrow \infty} \frac{S(X)}{X / 4} \geq 0.82
$$

In other words, the Erdôs conjecture is true for at least $82 \%$ of the integers $q \equiv 1(\bmod 4)$. Our method does not extend to show that the Erdôs conjecture is true for $100 \%$ of the moduli $q \equiv 1(\bmod 4)$. We examine this question briefly at the end of the paper. It seems to us that more ideas are needed to resolve the conjecture fully.

These questions have a long history beginning with Baker, Birch and Wirsing [Baker et al. 1973]. Their work was generalized by Gun, Murty and Rath [Gun et al. 2012] to the setting of algebraic number fields. The paper [Chatterjee and Murty 2014] gives new proofs of some of the background results of this area. We also refer the reader to [Tijdeman 2002] for an expanded survey of the early history.

\section{Notations and preliminaries}

From now onwards, we denote the field of rationals by $\mathbb{Q}$, the field of algebraic numbers by $\overline{\mathbb{Q}}$, Euler's totient function by $\varphi$ and Euler's constant by $\gamma$. We say a function $f$ is Erdősian modulo $q$ if $f$ is a periodic function with period $q$ and

$$
f(n)= \begin{cases} \pm 1 & \text { if } q \nmid n, \\ 0 & \text { otherwise }\end{cases}
$$


Also we will write $f(X) \lesssim g(X)$ to mean

$$
\limsup _{X \rightarrow \infty} \frac{f(X)}{g(X)} \leq 1
$$

Similarly, we write $f(x) \gtrsim g(x)$ to mean

$$
\liminf _{X \rightarrow \infty} \frac{f(X)}{g(X)} \geq 1
$$

\section{A. Okada's criterion.}

Proposition 2.1. Let the $q$-th cyclotomic polynomial $\Phi_{q}$ be irreducible over the field $\mathbb{Q}(f(1), \ldots, f(q))$. Let $M(q)$ be the set of positive integers which are composed of prime factors of $q$. For any integer $r$ and prime $p$, let $v_{p}(r)$ be the exponent of $p$ dividing $r$.

Then $L(1, f)=0$ if and only if the following conditions are satisfied:

$$
\begin{array}{ll}
\sum_{m \in M(q)} \frac{f(a m)}{m}=0 \quad \text { for every a with } 1 \leq a<q \text { and }(a, q)=1, \text { and } \\
\sum_{\substack{r=1 \\
(r, q)>1}}^{q} f(r) \epsilon(r, p)=0 & \text { for every prime divisor } p \text { of } q,
\end{array}
$$

where

$$
\epsilon(r, p)= \begin{cases}v_{p}(r) & \text { if } v_{p}(r)<v_{p}(q), \\ v_{p}(q)+\frac{1}{p-1} & \text { otherwise. }\end{cases}
$$

This proposition is a modification, due to Saradha and Tijdeman [2003], of a result of Okada [1986]. Note that Okada deduced the sufficient condition $2 \varphi(q)+1>q$ stated in the introduction from his original version of this criterion.

2B. Wirsing's theorem. The following proposition is due to Wirsing [1961].

Proposition 2.2. Let $f$ be a nonnegative multiplicative arithmetic function, satisfying

$$
\begin{aligned}
& |f(p)| \leq G \text { for all primes } p, \\
& \sum_{p \leq X} p^{-1} f(p) \log p \sim \tau \log X,
\end{aligned}
$$

with some constants $G>0, \tau>0$ and

$$
\sum_{p} \sum_{k \geq 2} p^{-k}\left|f\left(p^{k}\right)\right|<\infty
$$


if $0<\tau \leq 1$, then, in addition, the condition

$$
\sum_{p} \sum_{\substack{k \geq 2 \\ p^{k} \leq X}}\left|f\left(p^{k}\right)\right|=O(X / \log X)
$$

is assumed to hold. Then

$$
\sum_{n \leq X} f(n)=(1+o(1)) \frac{X}{\log X} \frac{e^{-\gamma \tau}}{\Gamma(\tau)} \prod_{p \leq X}\left(1+\frac{f(p)}{p}+\frac{f\left(p^{2}\right)}{p^{2}}+\cdots\right) .
$$

2C. Mertens' theorem. We also need a classical theorem of Mertens in a later section. We record the theorem here (see, for example, [Murty 2008, page 130]):

Proposition 2.3.

$$
\lim _{X \rightarrow \infty} \log X \prod_{p \leq X}\left(1-\frac{1}{p}\right)=e^{-\gamma} .
$$

\section{Exceptions to the conjecture of Erdôs}

We say that the Erdôs conjecture is false modulo $q$, if there is an Erdôsian function $f$ for which $L(1, f)=0$. The following proposition plays a fundamental role in our approach.

Proposition 3.1. If the Erdôs conjecture is false modulo $q$ with $q$ odd, then

$$
1 \leq \sum_{\substack{d \mid q \\ d \geq 3}} \frac{1}{\varphi(d)}
$$

Proof. By the hypothesis, there is an Erdôsian function $f(\bmod q)$ for which, we have $L(1, f)=0$. Applying Okada's criterion, we get

$$
\sum_{b \in M(q)} \frac{f(b)}{b}=0
$$

Let $d=(b, q)$, so that $b=d b_{1}$ with $\left(b_{1}, q / d\right)=1$. Then (2) can be written as

$$
-f(1)=\sum_{\substack{d \mid q \\ d \geq 3}} \frac{1}{d} \sum_{\substack{b_{1} \in M(q) \\\left(b_{1}, q / d\right)=1}} \frac{f\left(d b_{1}\right)}{b_{1}} .
$$

Taking absolute value of both sides, we get

$$
1 \leq \sum_{\substack{d \mid q \\ d \geq 3}} \frac{1}{d} \sum_{b_{1} \in M(d)} \frac{1}{b_{1}}
$$


Notice that the inner sum can be written as

$$
\sum_{b_{1} \in M(d)} \frac{1}{b_{1}}=\prod_{p \mid d}\left(1+\frac{1}{p}+\frac{1}{p^{2}}+\cdots\right)=\prod_{p \mid d}\left(1-\frac{1}{p}\right)^{-1}=\frac{d}{\varphi(d)} .
$$

Hence from (3), we get

$$
1 \leq \sum_{\substack{d \mid q \\ d \geq 3}} \frac{1}{\varphi(d)}
$$

Corollary 3.2. If $q$ is a prime power or a product of two distinct odd primes, then the Erdôs conjecture is true modulo q.

Proof. This is a pleasant elementary exercise.

Hence we have recovered the two basic cases of the conjecture which were given in the introduction, of course, also as a consequence of Okada's criterion.

Let $d(n)$ be the divisor function, that is, $d(n)$ is the number of divisors of $n$.

Corollary 3.3. If the smallest prime factor of $q$ is at least $d(q)$, then the Erdôs conjecture is true for $q$.

Proof. Let $l$ be the smallest prime factor of $q$. From the above proposition, if the Erdős conjecture is false modulo $q$, then we have

$$
\begin{aligned}
1 & \leq \sum_{\substack{d \mid q \\
d \geq 3}} \frac{1}{\varphi(d)} \\
& <\frac{1}{\varphi(l)} \sum_{\substack{d \mid q \\
d \geq 3}} 1=\frac{d(q)-2}{l-1},
\end{aligned}
$$

the strict inequality in the penultimate step coming from the fact that $q$ has at least two prime divisors. Thus, $l<d(q)$. Hence if $l \geq d(q)$, then the Erdôs conjecture is true modulo $q$.

Note that, Corollary 3.3 was not known previously. It implies that the conjecture is true for any squarefree number $q$ with $k$ prime factors, provided the smallest prime factor of $q$ is greater than $2^{k}$. Proposition 3.1 opens the door for a new approach to the study of Erdős's conjecture. Let us consider the following:

$$
S_{1}(X)=\mid\{q \equiv 1(\bmod 4), q \leq X \mid \text { Erdős conjecture is false modulo } q\} \mid \text {. }
$$


Then, we have

$$
\begin{aligned}
S_{1}(X) & \leq \sum_{\substack{q \leq X \\
q \equiv 1(\bmod 4)}} \sum_{\substack{d \mid q \\
d \geq 3}} \frac{1}{\varphi(d)} \leq \sum_{\substack{3 \leq d \leq X \\
d}} \frac{1}{\varphi(d)} \sum_{\substack{q \leq X \\
q \equiv 1(\bmod 4) \\
d \mid q}} 1 \\
& \leq \sum_{\substack{3 \leq d \leq X \\
d \text { odd }}} \frac{1}{\varphi(d)}\left(\frac{X}{4 d}+O(1)\right) \leq \sum_{\substack{3 \leq d \leq X \\
d \text { odd }}} \frac{1}{\varphi(d)} \frac{X}{4 d}+O\left(\sum_{3 \leq d \leq X} \frac{1}{\varphi(d)}\right) \\
& \leq \sum_{\substack{3 \leq d \leq X \\
d \text { odd }}} \frac{1}{\varphi(d)} \frac{X}{4 d}+O(\log X),
\end{aligned}
$$

where we have used the well-known fact that (see, for example, [Murty 2008, page 67])

$$
\sum_{d \leq X} \frac{1}{\varphi(d)}=O(\log X)
$$

Hence, we get

$$
\begin{aligned}
S_{1}(X) & \lesssim \frac{X}{4} \sum_{\substack{3 \leq d \\
d \text { odd }}} \frac{1}{d \varphi(d)} \\
& \lesssim \frac{X}{4}\left(\prod_{p \text { odd }}\left(1+\frac{1}{p \varphi(p)}+\frac{1}{p^{2} \varphi\left(p^{2}\right)}+\cdots\right)-1\right) \\
& \lesssim \frac{X}{4}\left(\prod_{p \text { odd }}\left(1+\frac{1}{p(p-1)}+\frac{1}{p^{3}(p-1)}+\cdots\right)-1\right) \\
& \lesssim \frac{X}{4}\left(\prod_{p \text { odd }}\left(1+\frac{1}{p(p-1)}\left(1+\frac{1}{p^{2}}+\frac{1}{p^{4}}+\cdots\right)\right)-1\right) \\
& \lesssim \frac{X}{4}\left(\prod_{p \text { odd }}\left(1+\frac{p}{(p-1)\left(p^{2}-1\right)}\right)-1\right) .
\end{aligned}
$$

The product is easily computed numerically and we have $S_{1}(X) \lesssim 0.33(X / 4)$. The following is an immediate corollary.

Corollary 3.4. $\mid\{q \equiv 1(\bmod 4), q \leq X \mid$ Erdôs conjecture is true for $q\} \mid \gtrsim 0.67 \frac{X}{4}$.

3A. Refinement using the second moment. By considering higher moments, we can improve the lower bound in the above corollary. We begin with the second moment. We include these estimates since they are of independent interest and self contained.

Proposition 3.5. $\mid\{q \equiv 1(\bmod 4), q \leq X \mid$ Erdôs conjecture is true for $q\} \mid \gtrsim 0.78 \frac{X}{4}$. 
Proof. Let us first consider the following inequality:

$$
\begin{aligned}
& S_{1}(X) \leq \sum_{\substack{q \leq X \\
q \equiv 1(\bmod 4)}}\left(\sum_{\substack{d \mid q \\
d \geq 3}} \frac{1}{\varphi(d)}\right)^{2} \\
& \leq \sum_{\substack{q \leq X \\
q \equiv 1(\bmod 4)}} \sum_{\substack{d_{1}\left|q, d_{2}\right| q \\
3 \leq d_{1}, d_{2}<q}} \frac{1}{\varphi\left(d_{1}\right) \varphi\left(d_{2}\right)} \\
& \leq \sum_{\substack{3 \leq d_{1}, d_{2} \leq X \\
d_{1}, d_{2} \text { odd }}} \frac{1}{\varphi\left(d_{1}\right) \varphi\left(d_{2}\right)} \sum_{\substack{q \leq X \\
q \equiv 1(\bmod 4) \\
d_{1}\left|q, d_{2}\right| q}} 1 \\
& \leq \sum_{\substack{3 \leq d_{1}, d_{2} \leq X \\
d_{1}, d_{2} \text { odd }}} \frac{1}{\varphi\left(d_{1}\right) \varphi\left(d_{2}\right)} \sum_{\substack{q \leq X \\
q \equiv 1(\bmod 4) \\
\left[d_{1}, d_{2}\right] \mid q}} 1 \\
& \leq \sum_{\substack{3 \leq d_{1}, d_{2} \leq X \\
d_{1}, d_{2} \text { odd }}} \frac{1}{\varphi\left(d_{1}\right) \varphi\left(d_{2}\right)}\left(\frac{X}{4\left[d_{1}, d_{2}\right]}+O(1)\right) .
\end{aligned}
$$

Hence, we have

$$
S_{1}(X) \leq \frac{X}{4} \sum_{\substack{3 \leq d_{1}, d_{2} \leq X \\ d_{1}, d_{2} \text { odd }}} \frac{1}{\varphi\left(d_{1}\right) \varphi\left(d_{2}\right)\left[d_{1}, d_{2}\right]}+O\left(\log ^{2} X\right) .
$$

By a simple numerical calculation, we deduce that

$$
S_{1}(X) \lesssim 0.22 \frac{X}{4} \text {. }
$$

Hence the conjecture holds for at least $78 \%$ of the positive integers congruent to $1(\bmod 4)$.

Similarly one can compute higher fractional moments to get an optimal result. For any $r>1$, we have

$$
S_{1}(X) \leq \sum_{\substack{q \leq X \\ q \equiv 1(\bmod 4)}}\left(\sum_{\substack{d \mid q \\ d \geq 3}} \frac{1}{\varphi(d)}\right)^{r} .
$$

We study this as a function of $r$. Using Maple we computed that the minimal value occurs at $r \sim 3.85^{1}$ and we get

$$
S_{1}(X) \lesssim 0.18 \frac{X}{4}
$$

\footnotetext{
${ }^{1}$ Code available at www.mast.queensu.ca/ murty/maplecode.pdf.
} 
Thus, we get $\mid\{q \equiv 1(\bmod 4), q \leq X \mid$ Erdős conjecture is true for $q\} \mid \gtrsim 0.82 \frac{X}{4}$, that is,

$$
\liminf _{X \rightarrow \infty} \frac{S(X)}{X / 4} \geq 0.82 .
$$

Hence, we have shown Theorem 1.1: the conjecture holds for at least $82 \%$ of the numbers congruent to $1(\bmod 4)$.

3B. An alternative approach. In this subsection, we discuss an alternative approach to this problem. It leads to a slightly weaker result. However this method is of independent interest, so we record it here. We begin with a further refinement of Proposition 3.1 by considering fractional moments there. From Proposition 3.1, if the Erdôs conjecture is false for odd $q$, then

$$
1 \leq \sum_{\substack{d \mid q \\ d \geq 3}} \frac{1}{\varphi(d)} .
$$

Adding 1 to both sides of the above inequality, we get

$$
2 \leq \sum_{d \mid q} \frac{1}{\varphi(d)},
$$

which can be rewritten as

$$
1 \leq \frac{1}{2} \sum_{d \mid q} \frac{1}{\varphi(d)} .
$$

Hence for any $\alpha>0$, Proposition 3.1 can be rewritten as follows.

Proposition 3.6. If Erdôs conjecture is false for odd $q$, then

$$
1 \leq \frac{1}{2^{\alpha}}\left(\sum_{d \mid q} \frac{1}{\varphi(d)}\right)^{\alpha} .
$$

As before, $S_{1}(X)=\mid\{q \equiv 1(\bmod 4), q \leq X \mid$ Erdôs conjecture is false for $q\} \mid$. Then from the above proposition, we get

$$
S_{1}(X) \leq \frac{1}{2^{\alpha}} \sum_{\substack{q \leq X \\ q \equiv 1(\bmod 4)}}\left(\sum_{d \mid q} \frac{1}{\varphi(d)}\right)^{\alpha} .
$$

Let $f_{\alpha}(q)=\left(\sum_{d \mid q} 1 / \varphi(d)\right)^{\alpha}$ and $\chi$ be the nontrivial Dirichlet character mod 4 . Then the above inequality becomes

$$
S_{1}(X) \leq \frac{1}{2^{\alpha+1}}\left(\sum_{\substack{q \leq X \\ q \text { odd }}} f_{\alpha}(q)+\sum_{\substack{q \leq X \\ q \text { odd }}} \chi(q) f_{\alpha}(q)\right) .
$$


Again, note that $f_{\alpha}(q)$ is a multiplicative arithmetic function. One can check that it also satisfies all the other hypotheses of Wirsing's theorem (Proposition 2.2) with $G=2^{\alpha}$ and $\tau=1$. So in light of Wirsing's theorem, we get

$$
\sum_{\substack{q \leq X \\ q \text { odd }}} f_{\alpha}(q) \sim X \frac{e^{-\gamma}}{\log X} \prod_{\substack{p \leq X \\ p \neq 2}}\left(1+\frac{f_{\alpha}(p)}{p}+\frac{f_{\alpha}\left(p^{2}\right)}{p^{2}}+\cdots\right)
$$

and

$$
\sum_{\substack{q \leq X \\ q \text { odd }}} \chi(q) f_{\alpha}(q) \sim X \frac{e^{-\gamma}}{\log X} \prod_{\substack{p \leq X \\ p \neq 2}}\left(1+\frac{\chi(p) f_{\alpha}(p)}{p}+\frac{\chi\left(p^{2}\right) f_{\alpha}\left(p^{2}\right)}{p^{2}}+\cdots\right) .
$$

Again, from Mertens theorem we know that

$$
\prod_{p \leq X}(1-1 / p) \sim \frac{e^{-\gamma}}{\log X}
$$

Hence we have

$$
\begin{aligned}
\sum_{\substack{q \leq X \\
q \text { odd }}} f_{\alpha}(q) & \sim \frac{X}{2} \prod_{\substack{p \leq X \\
p \neq 2}}(1-1 / p)\left(1+\frac{f_{\alpha}(p)}{p}+\frac{f_{\alpha}\left(p^{2}\right)}{p^{2}}+\cdots\right) \\
& \sim \frac{X}{2} p_{1} \text { (say) }
\end{aligned}
$$

and

$$
\begin{aligned}
\sum_{\substack{q \leq X \\
q \text { odd }}} \chi(q) f_{\alpha}(q) & \sim \frac{X}{2} \prod_{\substack{p \leq X \\
p \neq 2}}(1-1 / p)\left(1+\frac{\chi(p) f_{\alpha}(p)}{p}+\frac{\chi\left(p^{2}\right) f_{\alpha}\left(p^{2}\right)}{p^{2}}+\cdots\right) \\
& \sim \frac{X}{2} p_{2} \text { (say). }
\end{aligned}
$$

Now using the above two inequalities, (4) becomes

$$
S_{1}(X) \lesssim \frac{X}{2^{\alpha+2}}\left(p_{1}+p_{2}\right)
$$

Finally, using Maple ${ }^{2}$ we find that the quantity on the right hand side is minimized at $\alpha \sim 8.11$ and we get

$$
S_{1}(X) \lesssim 0.20 \frac{X}{4}
$$

\footnotetext{
${ }^{2}$ Code available at www.mast.queensu.ca/ murty/maplecode.pdf.
} 
Hence, we get

$$
\liminf _{X \rightarrow \infty} \frac{S(X)}{X / 4} \geq 0.80
$$

Remarks. One cannot hope to obtain $100 \%$ by these methods. In fact, one can show that there is a positive density (albeit small) of $q$ for which the inequality of Proposition 3.1 holds. Indeed, since

$$
\sum_{d \mid q} \frac{1}{\varphi(d)} \geq \prod_{p \mid q}\left(1+\frac{1}{p-1}\right)
$$

we can make the product (and hence the sum) arbitrarily large by ensuring that $q$ is divisible by all the primes in an initial segment. We can even ensure that these primes are congruent to $1(\bmod 4)$. We then take numbers which are divisible by this $q$ and congruent to $1(\bmod 4)$ and deduce that for all these numbers, the inequality in the proposition holds. Since the product on the right diverges slowly to infinity as we go through such numbers $q$, we obtain in this way a small density of numbers for which the inequality holds.

\section{Acknowledgements}

We thank Michael Roth for his help on using the Maple language as well as Sanoli Gun, Purusottam Rath and Ekata Saha for their comments on an earlier version of this paper. We also thank the referee for helpful comments that improved the quality of the paper.

\section{References}

[Baker et al. 1973] A. Baker, B. J. Birch, and E. A. Wirsing, "On a problem of Chowla", J. Number Theory 5 (1973), 224-236. MR 49 \#4959 Zbl 0267.10065

[Chatterjee and Murty 2014] T. Chatterjee and M. R. Murty, "Non-vanishing of Dirichlet series with periodic coefficients”, J. Number Theory 145 (2014), 1-21. MR 3253290

[Gun et al. 2012] S. Gun, M. R. Murty, and P. Rath, "Linear independence of Hurwitz zeta values and a theorem of Baker-Birch-Wirsing over number fields", Acta Arith. 155:3 (2012), 297-309. MR 2983455

[Livingston 1965] A. E. Livingston, "The series $\sum_{1}^{\infty} f(n) / n$ for periodic $f$ ", Canad. Math. Bull. 8 (1965), 413-432. MR 32 \#4104 Zbl 0129.02801

[Murty 2008] M. R. Murty, Problems in analytic number theory, Second ed., Graduate Texts in Mathematics 206, Springer, New York, 2008. Readings in Mathematics. MR 2008j:11001 Zbl 1190.11001

[Murty and Saradha 2007] M. R. Murty and N. Saradha, "Transcendental values of the digamma function”, J. Number Theory 125:2 (2007), 298-318. MR 2008g:11123 Zbl 1222.11097

[Murty and Saradha 2010] M. R. Murty and N. Saradha, "Euler-Lehmer constants and a conjecture of Erdös”, J. Number Theory 130:12 (2010), 2671-2682. MR 2011h:11078 
[Okada 1982] T. Okada, "On a certain infinite series for a periodic arithmetical function", Acta Arith. 40:2 (1982), 143-153. MR 83h:10099 Zbl 0402.10035

[Okada 1986] T. Okada, "Dirichlet series with periodic algebraic coefficients", J. London Math. Soc. (2) 33:1 (1986), 13-21. MR 87i:11087 Zbl 0589.10034

[Saradha and Tijdeman 2003] N. Saradha and R. Tijdeman, "On the transcendence of infinite sums of values of rational functions", J. London Math. Soc. (2) 67:3 (2003), 580-592. MR 2004b:11103 Zbl 1045.11051

[Tijdeman 2002] R. Tijdeman, "Some applications of Diophantine approximation", pp. 261-284 in Number theory for the millennium, III (Urbana, IL, 2000), A K Peters, Natick, MA, 2002. MR 2003j:11076 Zbl 1045.11022

[Wirsing 1961] E. Wirsing, "Das asymptotische Verhalten von Summen über multiplikative Funktionen”, Math. Ann. 143 (1961), 75-102. MR 24 \#A1241 Zbl 0104.04201

Received February 20, 2014. Revised November 5, 2014.

TAPAS CHATTERJEE

DEPARTMENT OF MATHEMATICS

INDIAN INSTITUTE OF TECHNOLOGY ROPAR

NANGAL ROAD

PUNJAB 140001

INDIA

tapasc@iitrpr.ac.in

M. RAM MURTY

DEPARTMENT OF MATHEMATICS \& STATISTICS

QUEEN'S UNIVERSITY

KINGSTON ON K7L3N6

CANADA

murty@mast.queensu.ca 


\title{
PACIFIC JOURNAL OF MATHEMATICS
}

\author{
msp.org/pjm
}

Founded in 1951 by E. F. Beckenbach (1906-1982) and F. Wolf (1904-1989)

\section{EDITORS}

Don Blasius (Managing Editor)

Department of Mathematics

University of California

Los Angeles, CA 90095-1555

blasius@math.ucla.edu

\author{
Paul Balmer \\ Department of Mathematics \\ University of California \\ Los Angeles, CA 90095-1555 \\ balmer@math.ucla.edu \\ Robert Finn \\ Department of Mathematics \\ Stanford University \\ Stanford, CA 94305-2125 \\ finn@math.stanford.edu \\ Sorin Popa \\ Department of Mathematics \\ University of California \\ Los Angeles, CA 90095-1555 \\ popa@math.ucla.edu
}

\author{
Vyjayanthi Chari \\ Department of Mathematics \\ University of California \\ Riverside, CA 92521-0135 \\ chari@math.ucr.edu \\ Kefeng Liu \\ Department of Mathematics \\ University of California \\ Los Angeles, CA 90095-1555 \\ liu@math.ucla.edu \\ Jie Qing \\ Department of Mathematics \\ University of California \\ Santa Cruz, CA 95064 \\ qing@ cats.ucsc.edu
}

\section{PRODUCTION}

Silvio Levy, Scientific Editor, production@msp.org

\section{SUPPORTING INSTITUTIONS}

ACADEMIA SINICA, TAIPEI

CALIFORNIA INST. OF TECHNOLOGY

INST. DE MATEMÁTICA PURA E APLICADA

KEIO UNIVERSITY

MATH. SCIENCES RESEARCH INSTITUTE

NEW MEXICO STATE UNIV.

OREGON STATE UNIV.

\author{
STANFORD UNIVERSITY \\ UNIV. OF BRITISH COLUMBIA \\ UNIV. OF CALIFORNIA, BERKELEY \\ UNIV. OF CALIFORNIA, DAVIS \\ UNIV. OF CALIFORNIA, LOS ANGELES \\ UNIV. OF CALIFORNIA, RIVERSIDE \\ UNIV. OF CALIFORNIA, SAN DIEGO \\ UNIV. OF CALIF., SANTA BARBARA
}

\author{
Daryl Cooper \\ Department of Mathematics \\ University of California \\ Santa Barbara, CA 93106-3080 \\ cooper@math.ucsb.edu \\ Jiang-Hua Lu \\ Department of Mathematics \\ The University of Hong Kong \\ Pokfulam Rd., Hong Kong \\ jhlu@maths.hku.hk \\ Paul Yang \\ Department of Mathematics \\ Princeton University \\ Princeton NJ 08544-1000 \\ yang@math.princeton.edu
}

These supporting institutions contribute to the cost of publication of this Journal, but they are not owners or publishers and have no responsibility for its contents or policies.

See inside back cover or msp.org/pjm for submission instructions.

The subscription price for 2015 is US \$420/year for the electronic version, and \$570/year for print and electronic.

Subscriptions, requests for back issues and changes of subscribers address should be sent to Pacific Journal of Mathematics, P.O. Box 4163, Berkeley, CA 94704-0163, U.S.A. The Pacific Journal of Mathematics is indexed by Mathematical Reviews, Zentralblatt MATH, PASCAL CNRS Index, Referativnyi Zhurnal, Current Mathematical Publications and Web of Knowledge (Science Citation Index).

The Pacific Journal of Mathematics (ISSN 0030-8730) at the University of California, c/o Department of Mathematics, 798 Evans Hall \#3840, Berkeley, CA 94720-3840, is published twelve times a year. Periodical rate postage paid at Berkeley, CA 94704, and additional mailing offices. POSTMASTER: send address changes to Pacific Journal of Mathematics, P.O. Box 4163, Berkeley, CA 94704-0163.

PJM peer review and production are managed by EditFLOW ${ }^{\circledR}$ from Mathematical Sciences Publishers.

\section{PUBLISHED BY}

\section{mathematical sciences publishers \\ nonprofit scientific publishing}

http://msp.org/

(C) 2015 Mathematical Sciences Publishers 


\section{PACIFIC JOURNAL OF MATHEMATICS}

Volume $275 \quad$ No. $1 \quad$ May 2015

Constant-speed ramps

OSCAR M. PERDOMO

Surfaces in $\mathbb{R}_{+}^{3}$ with the same Gaussian curvature induced by the

Euclidean and hyperbolic metrics

Nilton BARRoso and PEDRO RoITMAN

Cohomology of local systems on the moduli of principally polarized abelian surfaces

DAN PETERSEN

On certain dual $q$-integral equations

Ola A. Ashour, Mourad E. H. Ismail and Zeinab S.

MANSOUR

On a conjecture of Erdôs and certain Dirichlet series

TAPAS CHATTERJEE and M. RAM MURTY

Normal forms for CR singular codimension-two Levi-flat submanifolds

XiAnGHONG GONG and JiŘí LEBL

Measurements of Riemannian two-disks and two-spheres

FLORENT BALACHEFF

Harmonic maps from $\mathbb{C}^{n}$ to Kähler manifolds

JIANMING WAN

Eigenvarieties and invariant norms

Claus M. Sorensen

The Heegaard distances cover all nonnegative integers 